\title{
Performance of Phase Congruency and Linear Feature Extraction for Satellite Images Using Smoothing Algorithm
}

\author{
J.Krishna Chaithanya ${ }^{1}$, Babu.I $^{2}$, Shaik.Basha ${ }^{3}$ Dr.T.Ramashri ${ }^{4}$ \\ ${ }^{1,2,3}$ (Assistant Professor, Department Of ECE, Vardhaman College Of Engineering, Hyderabad, India.). \\ ${ }^{4}$ (Associate Professor, Department Of ECE, Sri Venkateswara University, Tirupati, A.P., India.)
}

\begin{abstract}
In computer vision all of the existing researches are interested in synthetic images features extraction. These images contain many types of features. Moreover, the satellite images are one most complex real image. Accordingly, many researches are developed in this way. The satellite images present a great variety of features due to the trouble what returns their treatment is little delicate. The automated extraction of linear features from remotely sensed imagery has been the subject of extensive research over several decades. Recent studies show promise for extraction of feature information for applications such as updating geographic information systems (GIS). Research has been stimulated by the increase in available imagery in recent years following the launch of several airborne and satellite sensors. In this paper, we introduce a new application of linear and phase congruency model for features extraction in satellite images. The aim of this paper is to exploit the advantages and the limitations of this model applied in satellite images features extraction. On the other hand, Adaptive minutia preserving smoothing algorithm used to improve the features extraction procedure. The paper also describes methods used for feature extraction and considers quantitative and qualitative accuracy assessment of these procedures.
\end{abstract}

Keywords: Satellite images, phase congruency model, smoothing algorithm, linear feature extraction.

\section{Introduction}

Humans have sought to extract information from imagery ever since the first photographic images were acquired. As early as the mid nineteenth century the French Army Corps of Engineers experimented with using aerial photographs for reconnaissance and mapping [1]. The expansion of photogrammetry and remote sensing have since been stimulated by advances such as the development of color film, the invention of the airplane, and unceasing improvement in instrumentation and techniques[1]. However, interest in feature extraction has increased significantly since the advent of digital imagery and the possibilities associated with electronic processing. Focused conferences provide an overview of many of the techniques available [2]. In addition, several commercially available photogrammetric workstation systems cited in the review by [3], now incorporate some automated feature extraction capability. Other companies such as [4] and [5] are developing software specifically targeted at feature extraction. However, since the majority of commercial vendors use proprietary algorithms this paper does not provide a comprehensive review of such systems. Within the field of feature extraction there are many areas of specialization. Some algorithms have been designed to identify specific target objects while others focus more generally on building or road extraction. The techniques for these different specializations can vary substantially. This paper focuses on the research performed for the purpose of extracting linear features from remotely sensed imagery. One of the primary reasons that researchers in the mapping sciences focus on linear feature recognition is because of the significance of roads in our society and the need to keep road locations updated [6]. [7], describe many of the challenges commonly associated with linear feature extraction and overview some common techniques.

Research into automated feature extraction from imagery dates back to the seventies. Since that time, technology has improved and commercial access to imagery has continued to expand.[8], described the improvements in feature extraction that were expected using 10 meter panchromatic imagery following the launch of the first SPOT satellite in 1986. In moderate resolution imagery, such as SPOT or Landsat Thematic Mapper (TM), linear features such as roads are often narrower than the spatial resolution of the satellite. [9], described this sub pixel problem as one of the complicating factors in extracting linear features using imagery from the satellite sensors available at that time. [10]Compared high spatial resolution aerial photography with SPOT and Landsat TM imagery for extracting road networks. Wang and Zhang [10] found that the success of linear feature extraction was particularly related to spatial resolution. Their experimentation found that photography out-performed the lower resolution satellite imagery when extracting roads in an urban environment. High spatial resolution imagery provides a detailed representation of road networks needed for many applications that cannot be obtained from lower resolution image sources [11]. In lower resolution imagery roads appear as curvilinear structures, while in higher resolution imagery roads appear as homogenous 
regions that satisfy certain shape or size constraints [12]. Roads are often characterized in high spatial resolution imagery as elongated regions [13].

The increased availability of high spatial resolution data has stimulated the development of techniques specifically targeted at taking advantage of the detail found in such imagery. For example, many of the techniques developed for road detection search for roads as pairs of edges: such techniques are unsuited to processing lower resolution imagery. Recently, Venkatesh and Owens [14] deal the scheme of the local energy model for features extraction. Phase congruency was further modified by Kovesi [2], [3] and extended to two dimensions over several orientations and combines this results in some way. In this paper, we introduce a new application of linear and phase congruency model for features extraction in satellite images. The aim of this paper is to exploit the advantages and the limitations of this model applied in satellite images features extraction.

\section{Linear Feature Extraction}

\section{System Design Model}

\section{Manual versus Automated Extraction}

Humans and computers have complementary strengths: humans are good at scanning large areas and recognizing objects, whereas computers are good at optimization, detailed delineation and repetition. Whether manual, automated, or a combination of the two, feature extraction can be a very involved process. Manual feature extraction harnesses the interpretation skills of the operator but can be time consuming and thus expensive to perform. With a growing body of digital data archived and a rapidly changing society, the efficient revision of cartographic databases implies some form of automated feature extraction.

Data capture is often the most expensive component in a GIS application and techniques developed that can alleviate this are welcomed. Automated systems offer the potential for time and labor savings and potentially may improve accuracy and consistency of plan metric data. The use of automated or semi-automated procedures can also provide cost savings by significantly reducing the training time of photo interpreters. Automated procedures may also have other less tangible benefits such as reducing operator fatigue. Humans have the ability to group simple features, such as points and lines, into meaningful structures. Semi-automated approaches rely on user provided cues to delineate road components. Only a short number of years ago considered fully automatic methods for feature extraction to be "far out of reach." Furthermore, the authors believe that using a semi-automated approach is optimal because humans perform identification almost flawlessly with limited effort. Humans are able to perceive shapes in noisy data and adapt to varying conditions, without being told explicitly what to expect. Writing computer code to simulate this ability is a significant challenge. Baumgartner, et al. (1999) found that their automatic road extraction was not absolutely reliable and generally required a human operator to edit the results. This typically entailed deleting wrongly extracted roads and inserting missing parts. Incorporating existing GIS data into the feature extraction process can reduce the need for human direction.

There is a wide range of techniques used to detect linear features in imagery. Many of the papers reviewed developed extraction techniques for locating a specific feature class, such as roads, while others considered adaptations to generalize the search to other linear features such as streams, railroads, and runways. The level of automation in the reviewed techniques varied significantly. Some procedures described in this paper require a significant amount of human input to select potential road locations.

\section{Mathematical Morphology model linear feature extraction}

The techniques of mathematical morphology have proven usefulin automating feature extraction. Mathematicalmorphology to search forroads in simulated and actual SPOT imagery, respectively.Dong (1997) used mathematical morphology to extract linearfeatures from gray scale aerial imagery.

In mathematical morphology, images are filtered using a kernel. The output of the filtering process depends on the match between the image and the kernel and the operation being performed. The two basic operations of mathematical morphology are dilation and erosion. The simplest example of mathematical morphology considers the analysis of binary images. The kernel typically used for binary imagery is a $3 \mathrm{X} 3$ array consisting of $1 \mathrm{~s}$, $0 \mathrm{~s}$, or $1 \mathrm{~s}$. Dilation and erosion can be performed on binary images using with the kernel shown in Figure 1. In dilation, the filter shown in Figure 1 is passed across the image with the resultant value for the center cell set to 1 if any 1 in the kernel matches a 1 in the image, which expands features in the image and closes any gaps. In erosion, a value of 1 is returned only if all the $1 \mathrm{~s}$ in the kernel are matched by $1 \mathrm{~s}$ in the image. Erosion shrinks image features and eliminates small features. Figure 2 shows a simple example where Figure $2 \mathrm{~b}$ and Figure 2c, respectively, illustrate dilation and erosion of Figure 2a. For ease of visualization, a value of 1 in Figure 2 is shown in the image as a black square and 0 as white. Dilation followed by erosion (closing) closes small gaps and connects sets; erosion followed by dilation (opening) removes small or narrow elements, without effecting large ones. 


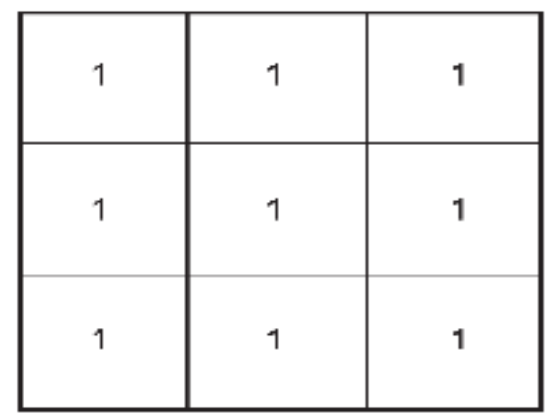

Figure 1. Basic kernel used for dilation and erosion in binary imagery.

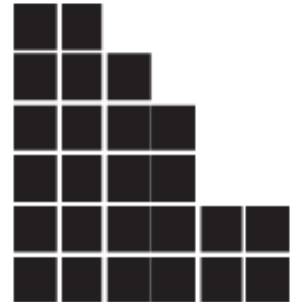

(a)

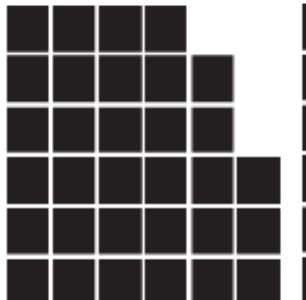

(b)

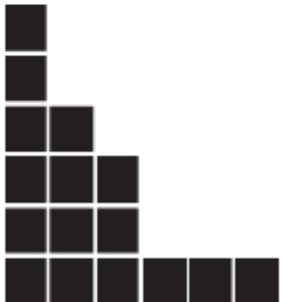

(c)

Figure 2. Simple example of erosion and dilation: (a) original (b) dilated (c) eroded.

\begin{tabular}{|l|l|l|}
\hline-1 & 1 & -1 \\
\hline-1 & 1 & -1 \\
\hline-1 & 1 & -1 \\
\hline
\end{tabular}

Figure 3. Directional kernel used for detecting linear features.

The directional filter shown in Figure 3 is used to identify linear features. Eroding an image using the kernel shown in Figure 3 and also eroding the image with rotations of the same kernel defines features with linear characteristics. When the rotations of the kernel shown in Figure 3 are applied to an area with homogenous intensity or a point feature, the output will be the same for all directions. Linear features, such as ridges and steps, are detected by identifying image locations that show variation in the output for different directional kernels.

Adaptive minutia preserving smoothing algorithm: To increase signal to noise ratio (SNR) and to stress on expectation characters, an improved adaptive minutia preserving smoothing algorithm is proposed using Matlab based on multi-scale and multidirectional masks. This algorithm keeps the mask's good performance in preserving details. It divides image into sub-images according to the statistics from image gradation-gradient histogram, and the adaptive threshold value generate according to the gradient information of the whole and the local image. This method deals with the difficulty of choosing threshold and improves the automation of image smoothing. The results of experiments show that the improved algorithm has more better performance than that classical algorithm either in reducing noise efficiently in intra-region and at edges or in keeping line-like structures such as edges and textures. 


\section{Multi-channel filtering}

\section{ThePhase Congruency Model}

Multi-channel filtering is popular methodology in imageanalysis. It employs a bank of filters with each filtermodeling a single channel. Several filtering functions suchas Gabor functions have been used to analyze the textimage and log Gabor functions have been used todetect edges.Image analysis entails the detection and extraction offeatures in an image, which are local changes in imageintensity such as lines and edges. An important category offeature extraction involves filtering a given image andmeasuring the filter response energy. There are many techniques proposed in the literature for analyzing satelliteimages. Here the multichannel filtering technique toextract satellite images features is used. In the local energy model, agiven image is filtered with a set of filters that haveidentical amplitude spectra but orthogonal phase spectra.The input satellite image is filtered with a bank of logGabor filters at various orientation and frequency witheach filter modeling a single channel.

The proposed approachwith the enormous amount of largely redundant data inimages, the extraction of images features is a fundamentalprocess of computer vision systems. In literature, severalapproaches are developed to extract $1 \mathrm{D}$ or 2D features.The local energy model has been used in manyapplications of image processing. It's relatively a newmodel for features extraction. Moreover, this modelpresents many advantages with other features detectionmethods.In the literature Venkatesh and Owensshowed that the local energy is proportional to phasecongruency, therefore local maxima of phase congruencycorrespond to local maxima in local energy. In practice,the local energy is used to detect features in images. According to Kovesi the phase congruency is definedBy

$$
P C(x)=\frac{\sum_{o} \sum_{n} W_{o}(x)\left\|_{\left[A_{n o}(x) \Delta \emptyset_{n o}(x)-T_{o}\right]}\right\|}{\sum_{o} \sum_{n} A_{n o}(x)+\varepsilon}
$$

Where $o$ and $n$ denotes the index over orientations and scales respectively, the symbols $I|l| l_{\text {denote the enclosed }}$ quantity is equal to itself when its value is positive, and zero otherwise. $A_{n o}(x)$ is the amplitude of the filter pair at position $x . \varepsilon$ is a very small positive real number, used to prevent division of zero, and its value is set to be 0.001 .

Satellite images present several false features due to external contributions (atmospheric noise). Indeed, in the weather domain many parameters like: rain, cloud and wind decrease the quality of the satellite images. Unfortunately, in the literature, all the existing approaches of features extraction suffer some drawbacks in the real images. So, the analysis of the satellite images requires a pre-processing like smoothing and the application of some techniques of thresholding.

Although the phase congruency model of images features makes no a priori assumption about the shape of image features, points of maximal phase congruency correspond to a wide range of features profiles. In this work, the features can be extracted are the step and the line edges which are local changes in image intensity. For these features: lines or edges, the Fourier phase spectrum is constant what returns their detection easy compared with other features. In addition, many other features can be identified in satellite images. An important category of feature extraction involves filtering a given image and measuring the filter response energy. There are many techniques proposed in the literature for analyzing texture. Here we use the multichannellog Gabor filtering technique to extract features in satellite images. Then, two smoothing algorithm are used to improve the quality of the satellite images. The proposed scheme for phase congruency computing is given by this diagram.

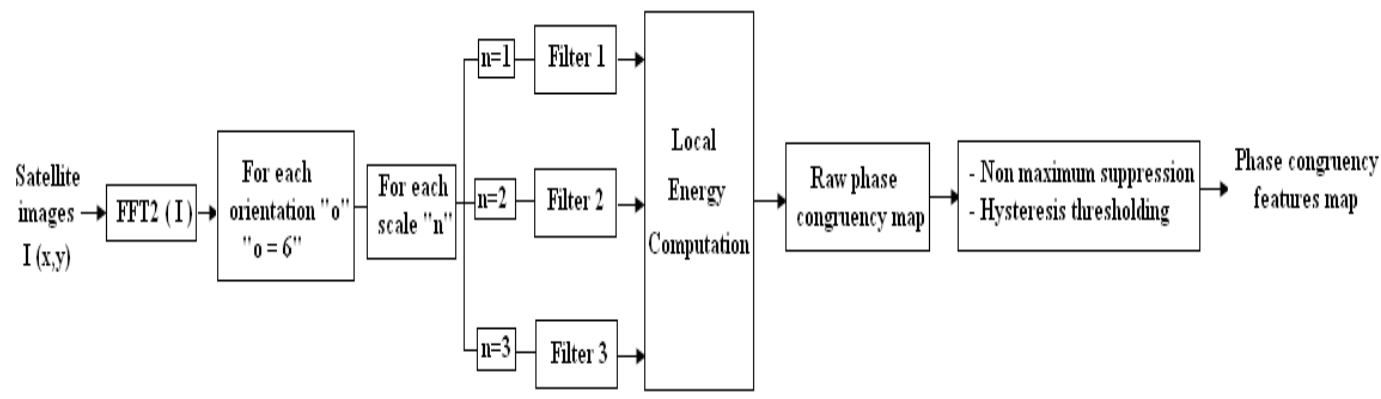

Fig. 4.The phase congruency computation scheme

\section{Comparison Between Linear AndPhase Congruency Models}

The phase congruency feature maps were obtained by performing nonmaximal suppression on the raw phase congruency images followed by hysteresis thresholding with upper and lower hysteresis threshold values fixed at phase congruency values. The hysteresis threshold values was set to [0.6-0.3] for satellite image 1 and [0.4-0.2] for the second and the third satellite images. The line and step features are detected and classified using 
the mean weight phase angle. Many of the techniques described require preliminary input from either a human operator or from existing data layers. One of the aims of ongoing research into feature extraction is to increase the level of automation while reducing the amount of human input required. To achieve this will require significant improvements in the reliability of the automated systems. Whether knowledge based computer systems will improve to the level that the interpreter is no longer required is yet to be seen.

Finally the paper describes that automatically extracting features from imagery increases the speed of data processing and improves the reliability of the parameters derived from these features. In terms of procedures provide significant benefits in terms of saving operator time and effort.

\section{Simulation Results}

Linear feature extractor is possible to see features that would not normally be visible and to locate geopositions of features that would otherwise be graphical. The level of brightness or reflectance of light from the surfaces in the image can be helpful with vegetation analysis, prospecting for minerals etc. Other usage examples include linear feature extraction, generation of processing work flows, and import/export of data for a wide variety of formats as shown in the figure above.
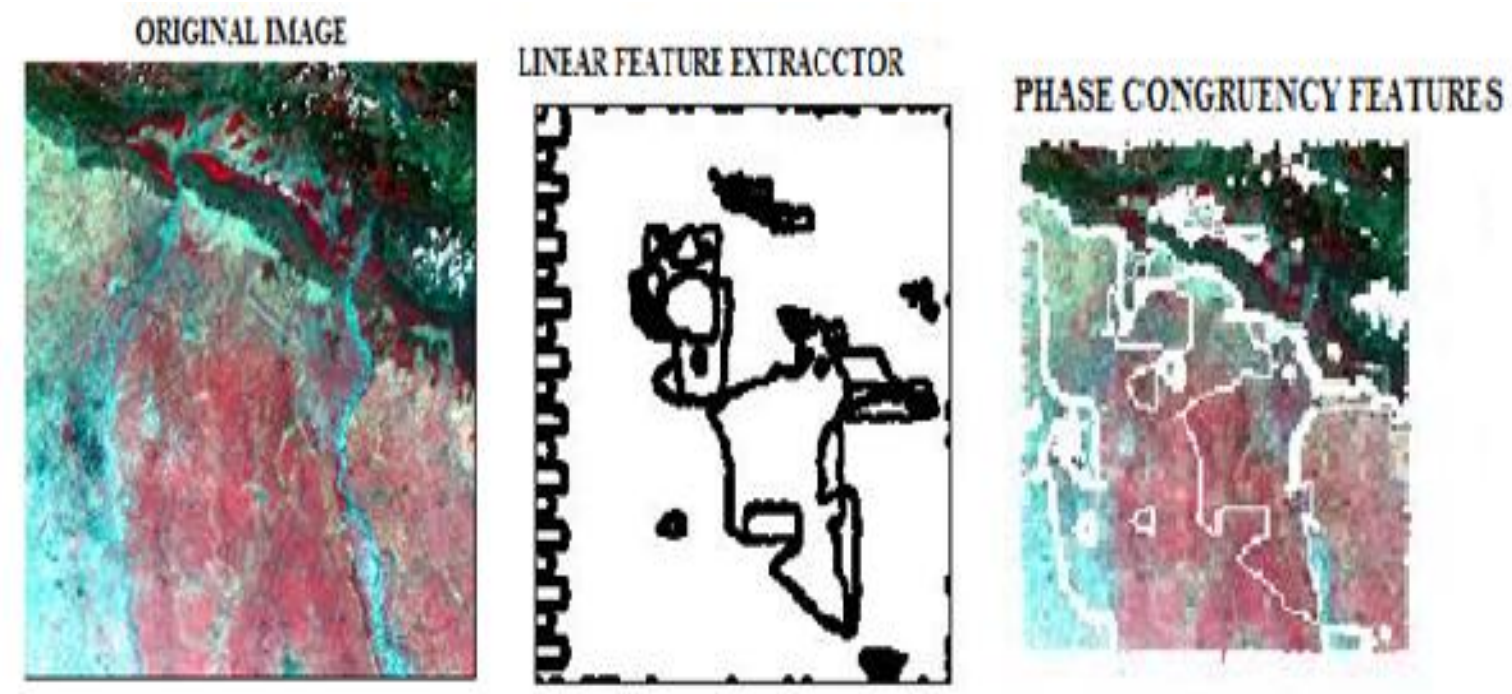

Figure 5: Experimental results of the satellite input image

The phase congruency feature maps were obtained by performing non maximal suppression on the raw phase congruency images followed by hysteresis thresholding with upper and lower hysteresis threshold values fixed at phase congruency values. In the above shows the phase congruency features map, the local energy map, the features images, features extracted, and histogram of feature type occurrence. These figures show that the satellite images have many false features.

\section{Conclusion}

Satellite images are a most complex images containing several types of features for that it analysis entails a preprocessing like smoothing, de-noising. With this method, the features extracted are the step and the line edges. Thus, the satellite images contain mainly these two linear features. This includes imagery from across the electromagnetic spectrum with a range of spatial resolutions. This paper reviewed a variety of techniques used for identifying linear, features in remotely-sensed imagery. The techniques reviewed relied on spectral, spatial, and radiometric rules in order to effectivelyextract features of interest

\section{References}

[1] Wolf, P.R., and B.A. Dewitt, 2000. Elements of Photogrammetry: WithApplications in GIS, Third Edition, McGraw Hill, Boston, Massachusetts, $608 \mathrm{p}$.

[2]. Baltsavias, E.P., A. Gruen, and L. Van Gool (editors), 2001. AutomatedExtraction of Man-Made Objects from Aerial and SpaceImages (III), A. A. Balkema Publishers, Lisse, The Netherlands, 415 p.

[3]. Plugers, P., 1999. Product survey on digital photogrammetric workstations, GIM International, 15(5/7):61-61/53.

[4]. Definiens, 2003. DefinienseCognition homepage, URL: http://www.definiens-imaging.com (last date accessed: 22 September 2004).

[5]. VLSI (Visual Learning Systems, Inc.), 2003. Feature Analyst home page, URL: http://www.featureanaly st.com (last date accessed: 22 September 2004). 
[6]. Pigeon, L., B. Moulin, B. Solaiman, T. Toutin, and K.P.B. Thomson, 1999a. Human-experts features extraction strategies for topographical map production and updating, Proceedings of International Geoscience and Remote Sensing Symposium, 28 June-02 July, Hamburg, Germany, pp. 296-298.

[7]. Park, J.S., R.A. Saleh, and Y. Yeu, 2002. Comprehensive survey of extraction techniques of linear features from remote sensing imagery for updating road spatial databases, Proceedings of the ACSM-ASPRS Annual Conference, 21-26 April, Washington D.C. (American Society for Photogrammetry and Remote Sensing), unpaginated CD-ROM.

[8]. Destival, I., 1986. Mathematical morphology applied to remote sensing,ActaAstronautica, 13(6/7):371-385.

[9]. Hemmer, T.H., 1996. Towards automation of the extraction of lines ofcommunication from multispectral images using a spatiospectralextraction technique, Proceedings of the SPIE: Algorithms forMultispectral and Hyperspectral Imagery II, 09-11 April, OrlandoFlorida, Volume 2758, pp. 115-126.

[10]. Wang, J., and Q. Zhang, 2000. Applicability of a gradient profile algorithm for road network extraction: Sensor, resolution and background considerations, Canadian Journal of Remote Sensing, 26(5):428-439.

[11]. Xiong, D., 2001. Automated road network extraction from high resolution images, Technical Notes, U.S. Department of Transportation, National Consortia on Remote Sensing in Transportation, Albuquerque, New Mexico, Issue 3, May, pp. 1-4.

[12], Hinz, S., A. Baumgartner, H. Mayer, C. Wiedemann, and H. Ebner, 2001. Automated Extraction of Man-Made Objects from Aerial and Space Images (III) (E. Baltsavias, A. Gruen and L. Van Gool, editors), A. A. Balkema Publishers, Lisse, The Netherlands, pp. 255-265.

[13]. Agouris, P., P. Doucette, and A. Stefanidis, 2001b. Spatiospectral cluster analysis of elongated regions in aerial imagery, Proceedings of the IEEE International Conference on Image Processing, 07-10 Oct, Thessaloniki, Greece, pp. 789-792.

[14] S. Venkatesh and R. A. Owens. "An energy feature detection scheme". In The International Conference on Image Processing, p. 553557, Singapore, 1989.

[15] P. D Kovesi. "Image features from phase congruency". Videre: Journal of Computer Vision Research 1, pp. 1-26, 1999

[16] P. D. Kovesi. "Invariant measures of image features from Phase Information". PhD thesis, The University of Western Australia, May 1996. 